\section{Shaker $\mathrm{K}^{+}$-channels are predicted to reduce the metabolic cost of neural information in Drosophila photoreceptors}

\author{
J. E. Niven ${ }^{1^{\star}}$, M. Vähäsöyrinki ${ }^{2}$ and M. Juusola ${ }^{1}$ \\ ${ }^{1}$ Physiological Laboratory, University of Cambridge, Downing Street, \\ Cambridge CB2 3EG, UK \\ ${ }^{2}$ Department of Physical Sciences, Division of Biophysics, \\ University of Oulu, 90041 Oulu Yliopisto, Finland \\ *Author for correspondence (jen22@hermes.cam.ac.uk).
}

Recd 21.01.03; Accptd 25.02.03; Online 08.04.03

Shaker $\mathbf{K}^{+}$-channels are one of several voltage-activated $\mathrm{K}^{+}$-channels expressed in Drosophila photoreceptors. We have shown recently that Shaker channels act as selective amplifiers, attenuating some signals while boosting others. Loss of these channels reduces the photoreceptor information capacity (bits $\mathrm{s}^{-1}$ ) and induces compensatory changes in photoreceptors enabling them to minimize the impact of this loss upon coding natural-like stimuli. Energy as well as coding is also an important consideration in understanding the role of ion channels in neural processing. Here, we use a simple circuit model that incorporates the major ion channels, pumps and exchangers of the photoreceptors to derive experimentally based estimates of the metabolic cost of neural information in wild-type (WT) and Shaker mutant photoreceptors. We show that in WT photoreceptors, which contain Shaker $\mathrm{K}^{+}$-channels, each bit of information costs approximately half the number of ATP molecules than each bit in Shaker photoreceptors, in which lack of the Shaker $\mathrm{K}^{+}$-channels is compensated by increased leak conductance. Additionally, using a Hodgkin-Huxley-type model coupled to the circuit model we show that the amount of leak present in both WT and Shaker photoreceptors is optimized to both maximize the available voltage range and minimize the metabolic cost.

Keywords: vision; photoreceptor; metabolic cost; information; Shaker; Drosophila

\section{INTRODUCTION}

Neurons contain an array of voltage-activated $\mathrm{K}^{+}$-channels that confer specific electrical properties upon their membranes enabling them to accurately process biologically relevant information (Rudy 1988; Weckström \& Laughlin 1995). To understand why particular channels are expressed in specific neurons requires detailed knowledge of their contribution to information processing (Hoffman et al. 1997; Niven et al. 2003). Additionally, since neural coding is metabolically expensive (Kety 1957; Ames 1997; Laughlin et al. 1998), the metabolic cost of a particular conductance may also be an important consideration.
The efficient expenditure of a limited energy budget is a major factor influencing all aspects of animal design; therefore, energy is thought to have been an important constraint upon the evolution of nervous systems (Laughlin 2001), including that of humans (Aiello \& Wheeler 1995). Potentially, energetic costs could limit absolute numbers of neurons and synaptic connections (information channels) in the brain (Laughlin et al. 1998), and influence the expression and distribution of ion channels and second messenger pathways in individual neurons. Indeed, the human brain uses energy at close to 20 W, accounting for $20 \%$ of the resting oxygen consumption (Kety 1957) of which over half is required to fuel ion pumps (Ames 1997).

The success of an individual also depends upon accurately determining and executing the appropriate behaviour. Nervous systems are, therefore, also constrained by the need to extract biologically germane, reliable information from the environment to generate such adaptive behaviour (De Polavieja 2002). The need for reliability, in the presence of noise, constrains information processing in nervous systems, setting lower limits for the numbers of neurons and synaptic connections (Laughlin et al. 1998) and influencing neuronal design.

In Drosophila photoreceptors both the amount of information (Juusola \& Hardie 2001; Niven et al. 2003), the information capacity (bits $\mathrm{s}^{-1}$ ), processed by a neuron and the metabolic cost of that information (ATP molecules per bit) (Laughlin et al. 1998) can be assessed with or without Shaker $\mathrm{K}^{+}$-channels. Photoreceptors lacking Shaker channels have a lower input resistance than wild-type (WT) photoreceptors, indicative of an increase in leak conductance (Niven et al. 2003). This increased leak conductance enables photoreceptors to maximize the spread of their signal over the available voltage range, which may also have a significant impact upon the cost of the information. Here, we use a circuit model of the photoreceptors based upon biophysical measurements to show that there is a dramatic increase (approximately twofold) in the metabolic cost per bit of information in photoreceptors that lack functional Shaker $\mathrm{K}^{+}$-channels.

\section{MATERIAL AND METHODS}

(a) Fly stocks

The WT strain was red-eyed Drosophila melanogaster Oregon Red. The Shaker channel mutation, $S h^{\mathrm{KS} 133}$ (a missense mutation in the core region resulting in non-functional Shaker channels; Kaplan \& Trout 1961; Salkoff \& Wyman 1981), was also expressed in red-eyed flies. Both fly strains were raised at $19^{\circ} \mathrm{C}$ in the dark.

\section{(b) Light stimulus}

Impaled photoreceptors were stimulated with repeated presentations of identical pseudorandom light contrast generated by a highintensity green light-emitting diode $\left(3 \times 10^{6}\right.$ photons s $^{-1}$; Marl Optosource).

\section{(c) Preparation and electrophysiology}

Flies were fixed using wax in a ceramic holder, and a small window was cut into the surface of the compound eye. Intracellular recordings were made using quartz microelectrodes, with resistances between 150 and $220 \mathrm{M} \Omega$, filled with $3 \mathrm{M} \mathrm{KCl}$. As an indifferent electrode a second blunt microelectrode filled with fly Ringer was also placed in the flies' head close to the eye. All recordings were made using an npi electronic amplifier in current-clamp mode. The temperature of the flies was maintained at $25^{\circ} \mathrm{C}$ throughout the experiments to within $1{ }^{\circ} \mathrm{C}$ accuracy using a custom-built thermocouple feeding back to a Peltier device. Photoreceptors were considered for analysis only if their dark resting potential was less than $-55 \mathrm{mV}$ and they had at least a $45 \mathrm{mV}$ saturating impulse response in dark-adapted conditions. Data acquisition, stimulus generation and signal analysis were performed by a purpose-built MATLAB interface. 
(a)

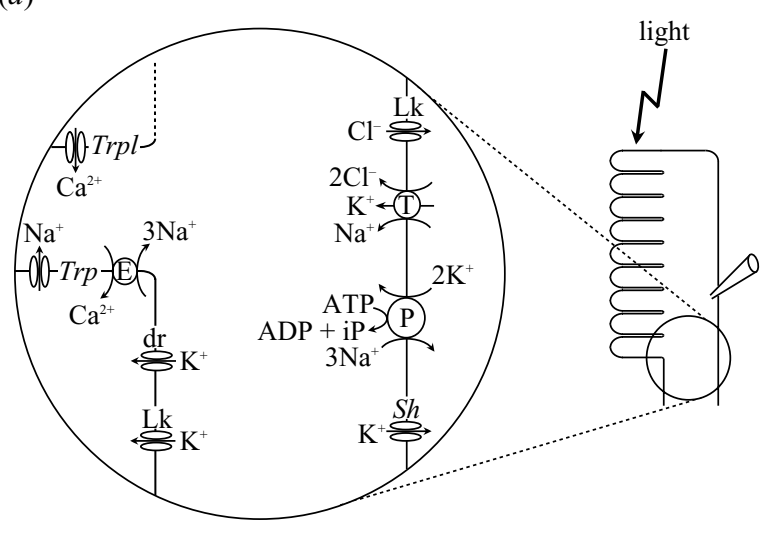

(b)(i)

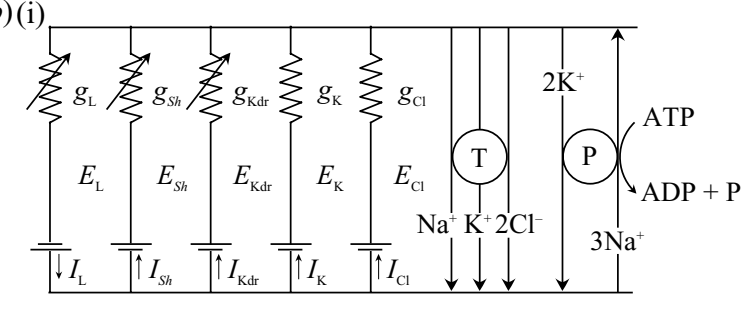

(ii)

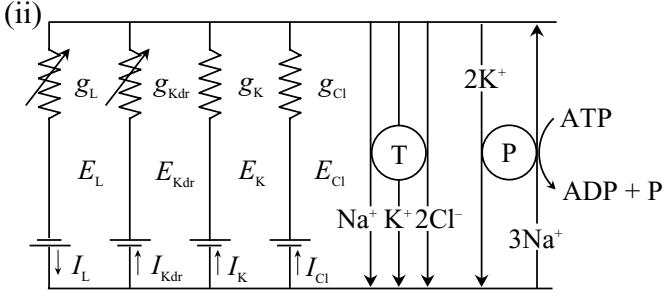

Figure 1. Ion channels, pumps and exchangers in the Drosophila compound eye. (a) A schematic diagram of part of a photoreceptor membrane showing the main ion channels, exchangers and pumps. (b) Electrical circuit models of (i) WT and (ii) Shaker photoreceptor membranes during light stimulation. Abbreviations: dr, delayed rectifier channel; Sh, Shaker channel; Lk, leak channel; Trp and Trpl, light-gated channels; $\mathrm{P}, \mathrm{Na}^{+} / \mathrm{K}^{+}$pump; $\mathrm{T}, \mathrm{Na}^{+} / \mathrm{K}^{+} / 2 \mathrm{Cl}^{-}$cotransporter; $E, 3 \mathrm{Na}^{+} / \mathrm{Ca}^{2+}$ exchanger.

(d) Calculation of the metabolic cost of information in wildtype and Shaker photoreceptors in steady-state conditions

Conductances for the circuit model of the photoreceptors were estimated by fitting a Hodgkin-Huxley model to current injection series recorded in vivo under dark- and light-adapted conditions (Niven et al. 2003). The circuit model contains Shaker and delayed rectifier conductances ( $g_{S h}$ and $g_{\mathrm{Kdr}}$, respectively), leak $\mathrm{Cl}^{-}$and $\mathrm{K}^{+}$ conductances ( $g_{\mathrm{Cllk}}$ and $g_{\mathrm{Klk}}$, respectively) and a light-gated conductance $\left(g_{\mathrm{L}}\right)$, as well as a $\mathrm{Na}^{+} / \mathrm{K}^{+}$pump. The activity of the $\mathrm{Na}^{+} / \mathrm{K}^{+}$ pump, the pump current $\left(I_{\mathrm{P}}\right)$, determines the cost of maintaining the internal $\mathrm{K}^{+}$concentration and external $\mathrm{Na}^{+}$concentration. A $\mathrm{Na}^{+} / \mathrm{K}^{+} / 2 \mathrm{Cl}^{-}$co-transporter, the probable $\mathrm{Cl}^{-}$accumulating mechanism in neurons (Delpire 2000), maintains the internal $\mathrm{Cl}^{-}$concentration. A fraction of the light conductance $(c a .40 \%)$ is carried by calcium ions $(20 \%$ of the light current), which are exchanged in a $1: 3$ ratio for sodium ions by the $\mathrm{Na}^{+} / \mathrm{Ca}^{2+}$ exchanger (figure 1) (Reuss et al. 1997; Oberwinkler \& Stavenga 2000).

Individual steady-state currents in either the light or dark were estimated from the known reversal potentials of individual ions and their conductances using Ohm's Law:

$I_{\mathrm{K} S h}=\left(E_{\mathrm{m}}-E_{\mathrm{K}}\right) g_{\mathrm{K} S h}$,

$I_{\mathrm{Kdr}}=\left(E_{\mathrm{m}}-E_{\mathrm{K}}\right) g_{\mathrm{Kdr}}$,

$I_{\mathrm{KIk}}=\left(E_{\mathrm{m}}-E_{\mathrm{K}}\right) g_{\mathrm{Klk}}$,

$I_{\mathrm{Cllk}}=\left(E_{\mathrm{m}}-E_{\mathrm{Cl}}\right) g_{\mathrm{Cllk}}$,

$I_{\mathrm{L}}=\left(E_{\mathrm{m}}-E_{\mathrm{L}}\right) g_{\mathrm{L}}$

where $E_{\mathrm{m}}$ is the resting potential, $E_{\mathrm{K}}$ is the potassium reversal potential, $E_{\mathrm{Cl}}$ is the chloride reversal potential and $E_{\mathrm{L}}$ is the reversal potential of the light-gated current. The $\mathrm{Na}^{+} / \mathrm{K}^{+}$pump hydrolyses one ATP molecule for every three $\mathrm{Na}^{+}$ions expelled and two $\mathrm{K}^{+}$ions imported. Thus, if the pump maintains the concentrations of these ions across the cell membrane,

$I_{\mathrm{P}}=\frac{1}{2}\left(I_{\mathrm{KSh}}+I_{\mathrm{Kdr}}+I_{\text {Kleak }}\right)-\frac{1}{4} I_{\text {Clleak }}$.

As the membrane potential is stable, the sum of all currents across the model membrane must equal zero:

$I_{\text {KSh }}+I_{\text {Kdr }}+I_{\text {Kleak }}+I_{\text {Clleak }}+I_{\mathrm{P}}+I_{\mathrm{L}}=0$.

The steady-state currents were adjusted to satisfy equation (2.7) using a simplex algorithm to minimize the changes for each current. Using these adjusted values the estimated number of ATP molecules hydrolysed per second can be calculated:

ATP molecules $\mathrm{s}^{-1}=I_{\mathrm{P}} N_{\mathrm{A}} / F$,

where $N_{\mathrm{A}}$ is Avogadro's constant and $F$ is Faraday's constant. The number of ATP molecules per bit of information was calculated by dividing the estimated number of ATP molecules hydrolysed per second by the measured information capacity, $C$ (bits ${ }^{-1}$ ):

ATP molecules per bit $=\frac{\left(I_{\mathrm{P}} N_{\mathrm{A}}\right) / F}{C}$.

\section{(e) Calculation of the metabolic cost of a natural-like} stimulus in a model photoreceptor

We drove a Hodgkin-Huxley-type model with a saturating naturallike conductance waveform to estimate the effect of varying levels of leak upon the available voltage range and the metabolic costs of both WT and $S h^{\mathrm{KS} 133}$ photoreceptors (see figure in electronic Appendix A available on The Royal Society's Publications Web site). This stimulus captures the essential features of a signal spreading over the available voltage range in WT and $S h^{\mathrm{KS} 133}$ photoreceptors (Niven et al. 2003); however, the absolute value for the voltage range is specific to the particular stimulus.

The voltage responses and individual conductances from the Hodgkin-Huxley model are first integrated and then divided by the simulation time to produce the average values. These values are used as inputs to an optimization model based upon the simplex algorithm, which minimizes the changes of voltage-dependent and leak conductances that are needed to fulfil equation (2.7). Each adjusted conductance is then multiplied by the corresponding driving force producing a set of currents. These currents are fed to the circuit model, which gives the metabolic cost as output. The new sets of conductances were used in the Hodgkin-Huxley model to resimulate the original traces to find out if the small changes owing to optimization would affect the responses: the resulting errors were not significant, which verified our method.

\section{RESULTS}

Drosophila photoreceptors contain voltage-activated $\mathrm{K}^{+}$channels including Shaker and slow-delayed rectifier (figure 1a; Hardie 1991; Hardie et al. 1991). By comparing the responses of WT to Shaker mutant ( $\left.S h^{\mathrm{KS} 133}\right)$ photoreceptors we estimated the contribution of the Shaker conductance to the cost of neural information. As the $\mathrm{Na}^{+} / \mathrm{K}^{+}$exchange pump is a major determinant of the metabolic cost of neural information (Laughlin et al. 1998; Attwell \& Laughlin 2001), voltage-activated and leak conductances may have significant effects upon these costs.

The estimated cost of maintaining the ionic gradients in both dark and light conditions (see $₫ 2$ ) was greater in Shaker than in WT photoreceptors (table 1). The bit cost 
Table 1. Properties of WT and Shaker photoreceptors.

(All values are mean \pm s.e., $n=21$ for WT values and $n=14$ for $S h$ values unless otherwise stated. Estimates from the models are quoted as single values.)

\begin{tabular}{lcccc}
\hline & WT, dark & WT, light & $S h^{\mathrm{KS} 133}$, dark & $S h^{\mathrm{KS} 133}$, light \\
\hline membrane properties & & & & \\
$\quad$ membrane potential, $E_{\mathrm{m}}(\mathrm{mV})$ & $-68.1 \pm 3.2$ & $-42.9 \pm 4.1$ & $-64.3 \pm 5.4$ & $-45.2 \pm 6.5$ \\
$\quad$ steady-state resistance $(\mathrm{M} \Omega)$ & $410.5 \pm 34.5$ & $216 \pm 22.4^{\mathrm{a}}$ & $225.4 \pm 25.6$ & $158 \pm 17.7^{\mathrm{a}}$ \\
information capacity $\left(\mathrm{bits}^{-1}\right)$ & - & $217.9 \pm 43.3$ & - & $138.9 \pm 8.4$ \\
cost $\left(10^{7}\right.$ ATP molecules s $\left.{ }^{-1}\right)$ & 3.80 & 20.98 & 7.98 & 27.06 \\
bit cost $\left(10^{6}\right.$ ATP molecules per bit) & - & 0.96 & - & 1.95 \\
\hline
\end{tabular}

${ }^{\mathrm{a}} n=8$.

(ATP per bit) was obtained by dividing the rate of ATP hydrolysis (ATP s ${ }^{-1}$ ) by the information capacity (bits s ${ }^{-1}$ ) (see $₫ 2$ ). We have previously shown that the information capacity of WT photoreceptors is ca. 220 bits s $^{-1}$ whereas that of Shaker photoreceptors is ca.140 bits s ${ }^{-1}$ (Niven et al. 2003). The increased rate of ATP hydrolysis in Shaker photoreceptors coupled with their reduced information capacity led to an approximately twofold increase in their bit cost when compared with WT photoreceptors (table 1). Several factors may lead us to underestimate the bit cost for Drosophila photoreceptors during natural stimuli including lower information transmission rates and the energy required for early steps in phototransduction (e.g. phosphorylation or amplification; Laughlin et al. 1998; Hardie \& Raghu 2001). However, similar calculations for Calliphora photoreceptors (Laughlin et al. 1998) produce estimates of metabolic cost close to the experimentally determined values (Hamdorf et al. 1988), indicating that our estimates of the bit cost are valid.

The increased bit cost in $S h^{\mathrm{KS} 133}$ photoreceptors is due to an increase in leak conductance to compensate for the loss of the Shaker conductance. The amount of compensatory leak in $S h^{\mathrm{KS} 133}$ and WT photoreceptors maximizes their available voltage range, increasing the number of possible states for information transmission (Niven et al. 2003). Metabolic cost may also be an important factor affecting the amount of leak conductance. To determine this we drove the model with a natural-like dynamic stimulus and calculated the metabolic costs with the circuit model (see $\ 2$ ). Both $S h^{\mathrm{KS} 133}$ and WT photoreceptors were optimized in terms of metabolic cost and available voltage range (figure 2); however, WT photoreceptors incurred a lower metabolic cost than those of $\mathrm{Sh}^{\mathrm{KS} 133}$ owing to the Shaker conductance.

\section{DISCUSSION}

Evidence is accumulating that nervous systems optimize coding and information transmission to minimize the amount of energy expended upon neural processing (Laughlin 2001). Previous studies had estimated the metabolic cost of neural information processing but had not experimentally examined the roles of individual cellular components, such as ion channels, to these costs (Laughlin et al. 1998; Attwell \& Laughlin 2001). We have shown that the loss of the Shaker $\mathrm{K}^{+}$-conductance increases the metabolic cost of information in Drosophila photoreceptors. This increase is due to the Shaker conductance that is present at resting potential but which rapidly

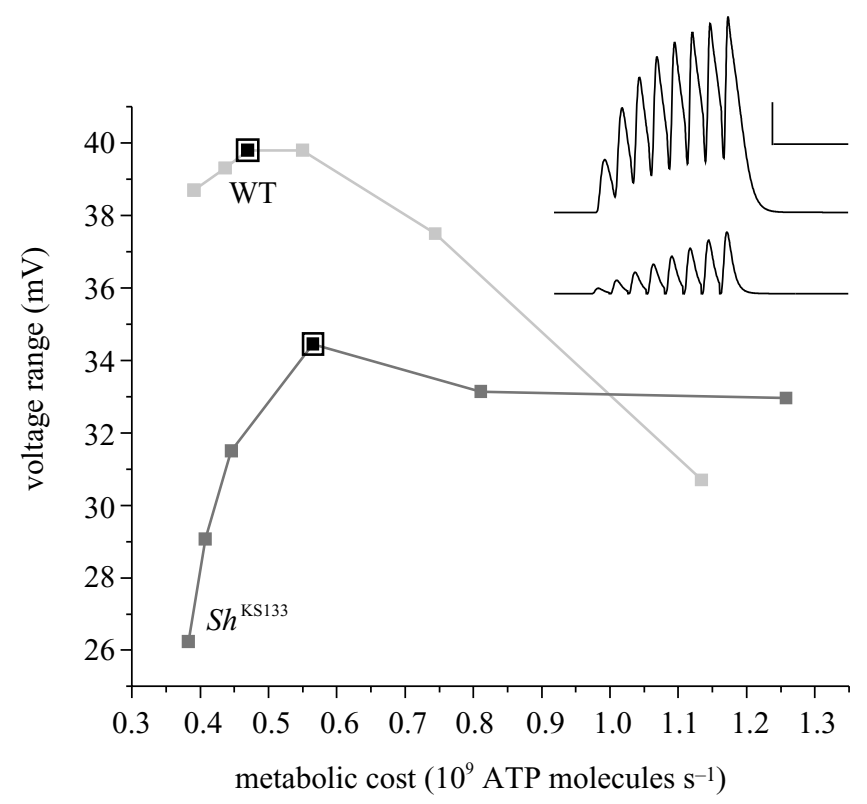

Figure 2. The size of leak conductance optimizes the voltage range of the WT and $S h^{\mathrm{KS} 133}$ photoreceptors in terms of metabolic cost. Each hypothetical membrane contained more or less leak conductance than the experimentally determined values. The range was taken as the difference between the minimum and maximum peak voltage responses to the conductance stimulus. The size of the leak conductance in both the WT (grey) and Shaker (dark grey) models affects the size of the voltage range. The experimentally determined values for the WT and $S h^{\mathrm{KS} 133}$ photoreceptors (black squares with surround) maximize the available voltage range. Inset, responses of the WT model membrane (upper) to a series of lognormal conductance pulses (lower). Horizontal scale bar, $200 \mathrm{~ms}$; vertical scale bar, $10 \mathrm{mV}$ (upper) and $0.8 \mathrm{mS} \mathrm{cm} \mathrm{cm}^{-2}$ (lower).

turns off when photoreceptors are depolarized (owing to the inactivation of the Shaker channels). Thus, WT photoreceptors reduce their membrane resistance without paying the metabolic cost at high light intensities when the membrane potential is far from the potassium reversal potential. In $S h^{\mathrm{KS} 133}$ flies the compensatory leak conductance does not inactivate, increasing the amount of work the $\mathrm{Na}^{+} / \mathrm{K}^{+}$pump must do at high light intensities and, consequently, increasing the metabolic cost of information.

Understanding the relationship between individual conductances and the metabolic cost of information may also elucidate the function of other components in neural sig- 
nalling such as neuromodulators. For example, serotonin positively shifts the voltage range of Shaker and delayed rectifier $\mathrm{K}^{+}$-channels in Drosophila photoreceptors so that they are completely inactive at rest (Hevers \& Hardie 1995). This would remove a large proportion of the $\mathrm{K}^{+}$ conductance in the WT photoreceptors in the dark, significantly reducing the metabolic cost of maintaining the resting potential. However, the shifted voltage range would increase the metabolic cost of information at high light intensities, suggesting that serotonin should be released at night to minimize metabolic cost. Thus, metabolic cost may be an important factor not only in determining which combinations of ion channels are expressed in particular neurons, but also in the plasticity within the properties of these ion channels.

\section{Acknowledgements}

The authors thank S. B. Laughlin, R. C. Hardie and M. Weckström for valuable discussions and comments on this manuscript. This work was supported by the Wellcome Trust (J.E.N. and M.J.), The Royal Society and BBSRC (M.J.) and the FGSN (M.V.).

Aiello, L. C. \& Wheeler, P. 1995 The expensive tissue hypothesis: the brain and the digestive system in human and primate evolution. Curr. Anthropol. 36, 199-221.

Ames, A. 1997 Energy requirements of brain function: when is energy limiting? In Mitochondria and free radicals in neurodegenerative disease (ed. M. F. Beal, N. Howell \& I. Bodis-Wollner), pp. 17-27. New York: Wiley-Liss.

Attwell, D. \& Laughlin, S. B. 2001 An energy budget for signaling in the grey matter of the brain. F. Cereb. Blood Flow Metab. 21, 1133-1145.

De Polavieja, G. G. 2002 Errors drive the evolution of biological signaling to costly codes. F. Theor. Biol. 214, 657-664.

Delpire, E. 2000 Cation-chloride cotransporters in neuronal communication. News Physiol. Sci. 15, 309-312.

Hamdorf, K., Hochstrate, P., Hoglund, G., Burbach, B. \& Wiegand, U. 1988 Light activation of the sodium-pump in blowfly photoreceptors. F. Comp. Physiol. A 162, 285-300.

Hardie, R. C. 1991 Voltage-sensitive potassium channels in Drosophila photoreceptors. F. Neurosci. 11, 3079-3095.
Hardie, R. C. \& Raghu, P. 2001 Visual transduction in Drosophila. Nature 413, 186-193.

Hardie, R. C., Voss, D., Pongs, O. \& Laughlin, S. B. 1991 Novel potassium channels encoded by the Shaker gene in Drosophila photoreceptors. Neuron 6, 477-486.

Hevers, W. \& Hardie, R. C. 1995 Serotonin modulates the voltage dependence of delayed rectifier and Shaker potassium channels in Drosophila photoreceptors. Neuron 14, 845-856.

Hoffman, D. A., Magee, J. C., Colbert, C. M. \& Johnston, D. 1997 $\mathrm{K}^{+}$channel regulation of signal propagation in dendrites of hippocampal pyramidial neurons. Nature 387, 869-875.

Juusola, M. \& Hardie, R. C. 2001 Light adaptation in Drosophila photoreceptors: I. Response dynamics and signalling efficiency at $25^{\circ}$ C. F. Gen. Physiol. 117, 3-25.

Kaplan, W. D. \& Trout, W. E. 1961 The behaviour of four neurological mutants of Drosophila. Genetics 61, 399-409.

Kety, S. S. 1957 The general metabolism of the brain in vivo. In Metabolism of the nervous system (ed. D. Richter), pp. 221-237. London: Pergamon.

Laughlin, S. B. 2001 Energy as a constraint on the coding and processing of sensory information. Curr. Opin. Neurobiol. 11, 475-480.

Laughlin, S. B., de Ruyter Van Steveninck, R. R. \& Anderson, J. C. 1998 The metabolic cost of neural information. Nature Neurosci. $1,36-41$.

Niven, J. E., Vähäsöyrinki, M., Kauranen, M., Hardie, R. C., Juusola, M. \& Weckström, M. 2003 The contribution of Shaker $\mathrm{K}^{+}$-channels to the information capacity of Drosophila photoreceptors. Nature 421, 630-634.

Oberwinkler, J. \& Stavenga, D. G. 2000 Calcium imaging demonstrates colocalization of calcium influx and extrusion in fly photoreceptors. Proc. Natl Acad. Sci. USA 97, 8578-8583.

Reuss, H., Mojet, M. H., Chyb, S. \& Hardie, R. C. 1997 In vivo analysis of the light-sensitive channels, TRP and TRPL. Neuron 19, 1249-1259.

Rudy, B. 1988 Diversity and ubiquity of $\mathrm{K}^{+}$channels. Neuroscience 25, 729-749.

Salkoff, L. \& Wyman, R. 1981 Genetic modification of potassium channels in Drosophila Shaker mutants. Nature 293, 228-230.

Weckström, M. \& Laughlin, S. B. 1995 Visual ecology and voltagegated ion channels in insect photoreceptors. Trends Neurosci. 18, $17-21$.

Visit http://www.pubs.royalsoc.ac.uk to see an electronic appendix to this paper. 